\title{
SYNCHROTRON RADIATION MONITORING SYSTEM AT BEPC
}

\author{
L.Ma, Z.Zhao, D.Liu, J.Cao, L.Wang, K.Xue, S.Wang and H.Yu \\ Institute of High Energy Physics, Beijing 100039, China
}

\section{Abstract}

The synchrotron radiation by the relativistic particles is a useful and important diagnostic tool to obtain various beam parameters. The visible light of the synchrotron radiation emitted in a bending magnet of the BEPC storage ring is reflected through the window by a watercooled mirror in the vacuum chamber and then directed by a remote controlled mirror onto the optical bench supporting the diagnostic instruments in the dark room. These instruments include a CCD camera providing the image of the beam transverse profile on a TV monitor, a lens system focusing the synchrotron light onto a solid state scanned photodiode arrays to obtain the beam height or width, a streak camera to measure the bunch length for a single pass, and a two-dimensional position sensing photo-detector to monitoring the drift of the beam position. This paper will give the detailed description of the synchrotron radiation monitoring system at BEPC.

\section{GENERAL DESCRIPTION}

The Beijing Electron-Positron Collider (BEPC) was built for the high energy physics experiment and the synchrotron radiation research. The BEPC has been in operation for around 10 years. The synchrotron radiation monitoring system has been put into operation since the startup of the BEPC. The characteristics of the electron and positron beams circulating in the ring, such as the transverse beam size, the bunch length and the beam position, can be measured with the system. Table 1 lists the designing values of some parameters related with the synchrotron radiation monitoring system. The vertical beam size in the table assumes an emittance coupling of 3\%.

Table 1: Some parameters related with the synchrotron radiation monitoring system of BEPC

\begin{tabular}{|l|c|c|}
\hline Parameters & Collid. mode & SR mode \\
\hline Ring energy (GeV) & $1.55-2.0$ & 2.2 \\
\hline Revolution freq. (MHz) & \multicolumn{2}{|c|}{1.247} \\
\hline Natural emitt. (nm-rad) & $406-666$ & 76 \\
\hline $\begin{array}{l}\text { H/V rms beam size at } \\
\text { source point (mm) }\end{array}$ & $\begin{array}{c}1.32 / 0.42- \\
1.69 / 0.54\end{array}$ & $0.58 / 0.26$ \\
\hline Rms bunch length (ps) & $360-470$ & 255 \\
\hline $\begin{array}{l}\text { Bending radius at } \\
\text { source point (m) }\end{array}$ & \multicolumn{2}{|c|}{10.34} \\
\hline $\begin{array}{l}\text { Distance from source } \\
\text { point to first mirror (m) }\end{array}$ & \multicolumn{2}{|c|}{1.75} \\
\hline $\begin{array}{l}\text { Natural open angle of } \\
\text { light, } \lambda=491 \text { nm (mrad) }\end{array}$ & \multicolumn{2}{|c|}{2.2} \\
\hline
\end{tabular}

Two BEPC primary flat and optically polished mirrors in the vacuum chamber, one each for the positron and electron beams, are made of $\mathrm{Cu}$ coasting with gold. The visible part of the synchrotron light is reflected horizontally through the side window by the water-cooled mirror and then directed vertically by a remote controllable mirror to the optical building where contains an optical table, detector electronics, work space. The third mirror brings the light into the horizontal plane again and to the optical table supporting the optical components comprising the detector system. The newly-built optical table was put into use at the beginning of 1998. The surface area of the optical table is $1.81 \times 1.2 \mathrm{~m}^{2}$ and is more than 5 times of the area of the original optical table.

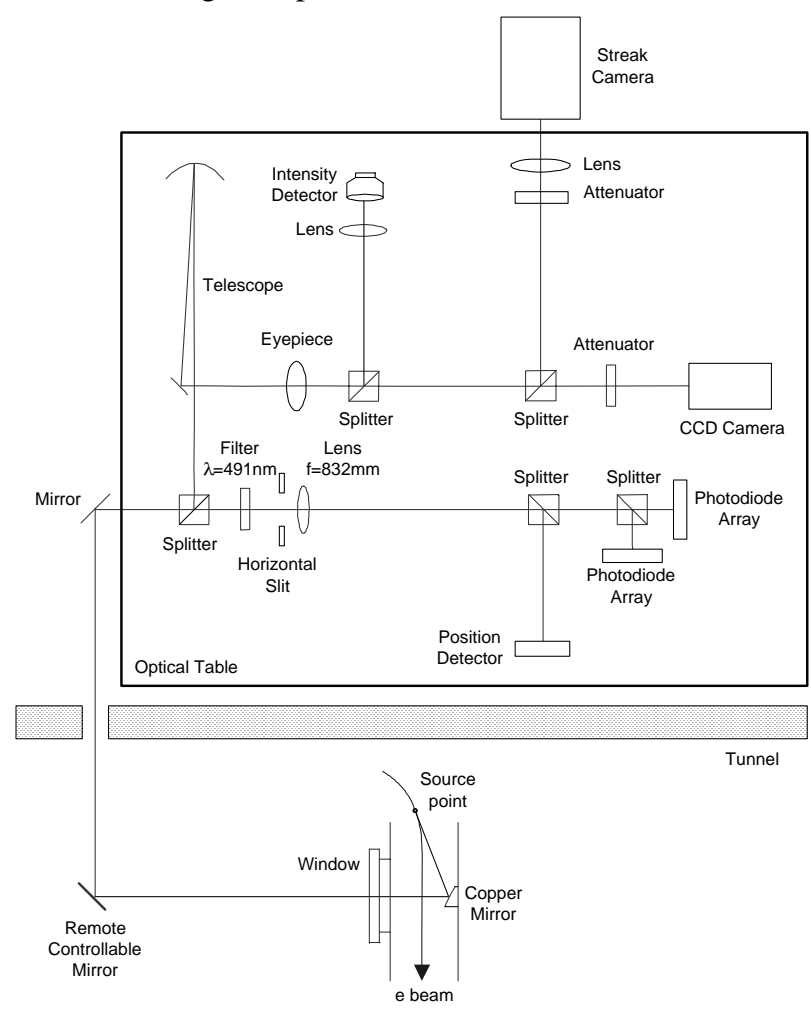

Figure 1: Setup of the BEPC synchrotron radiation monitoring system

Figure 1 shows the setup of the synchrotron radiation monitoring system at BEPC. As can be seen from the figure, a CCD camera provides the beam profile on a TV monitor. The image of the beam profile is also displayed in the center control room. The beam height and width are measured with a lens system focusing the synchrotron light onto two solid state scanned photodiode arrays. The bunch length for a single pass is measured with a streak camera only for the electron beam. The light beam posi- 
tion is monitored with a position sensing photo-detector. Figure 2 shows a photo of the new optical table.

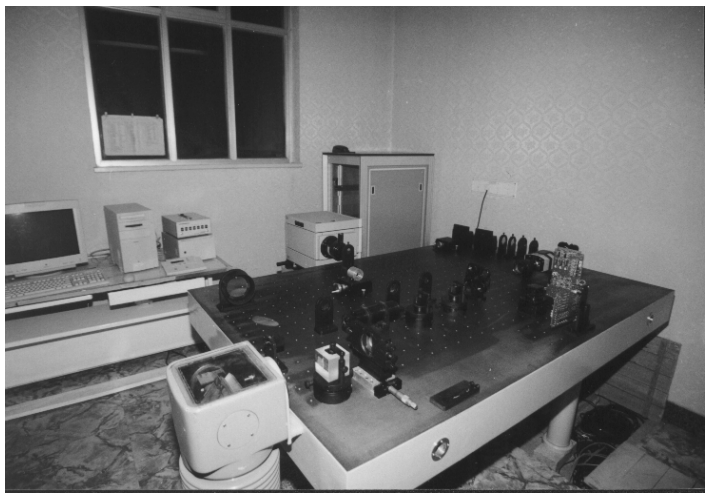

Figure 2: New optical table for the $\mathrm{e}^{-}$beam

\section{PHOTODIODE ARRAY}

Two solid state self-scanning linear photodiode arrays of EG\&G Reticon, Model RL0512S, have been used as the detectors for the transverse beam size measurements, one each for the horizontal and vertical planes. The array consists of a row of silicon photodiodes, each with an associated storage capacitor on which to integrate photocurrent and a multiplex switch for periodic readout via an integrated shift register scanning circuit. The photodiode array contains 512 diode sensor elements of $25 \mu \mathrm{m}$ centers, so the overall length of the array is $12.8 \mathrm{~mm}$. The width of each sensor element is $2.5 \mathrm{~mm}$ giving each element a geometry with the 100:1 aspect ratio. The spectral response range for the quartz-windowed array is from $250-1000 \mathrm{~nm}$ with a peak response around $750 \mathrm{~nm}$.

The synchrotron light with $\lambda=491 \mathrm{~nm}$ collected on a sensor area generates a charge which is proportional to the intensity of the incident light and to the integration period. Charges accumulated on the photodiodes are readout sequentially and processed by a sample and hold signal processing circuit. The resulting video output signal is send to an oscilloscope and appears the sampled and held boxer waveform. The FWHM width of the waveform is then read by a PC with the GPIB interface card to obtain the rms half height and width of the beam.

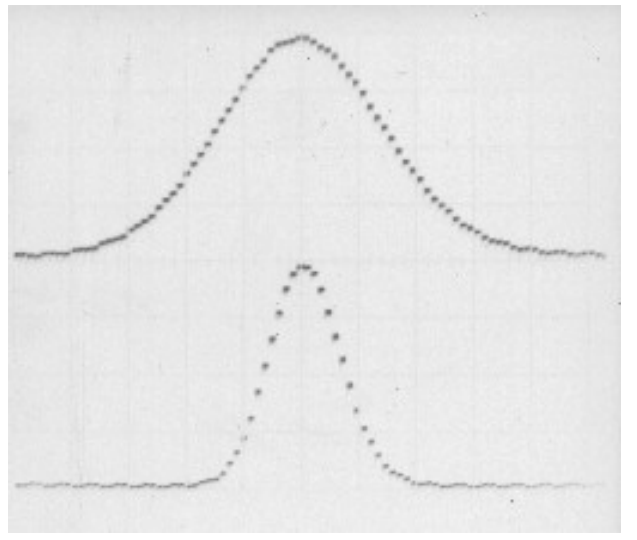

Figure 3: Horizontal (upper trace) and vertical (lower trace) beam profiles
It was decided to use a single focusing lens system for both transverse beam size and beam position measurements. The focus length and the diameter of the lens are $832 \mathrm{~mm}$ and $80 \mathrm{~mm}$, respectively. Considering the object distance of $6800 \mathrm{~mm}$ and image distance of $948 \mathrm{~mm}$, the magnification coefficient of the optics is 0.139 .

Figure 3 shows a photo of the oscilloscope waveforms of beam profiles at $1.55 \mathrm{GeV}$. Taking account of the magnification 0.139 of the optics and various measurement errors such as curvature of the electron beam orbit, field depth and diffraction errors, the horizontal and vertical rms beam size at the source point can be derived.

\section{STREAK CAMERA}

A streak camera Model C5680-11 of the Hamamatsu Photonics has been used for the bunch length measurement since 1996. The streak camera can be divided into the main unit C5680, the synchroscan unit M5675 and the dual time axis expansion units M5679. The C5680 includes the input optics, the photocathode, the MCP, the CCD camera, and other image control system. The M5675 controls the vertical sweep by the signal synchronized to the incident light. It insures the image section of the device to keep up with the high-speed light, and also insures the high temporal resolution within 2 ps. The M5679 controls the horizontal sweep of the screen image and determines the acquisition time of every picture. The whole system also includes the delay unit C1097, a remote controller, a monitor, a pulse generator, a control computer and other auxiliary parts as shown in Figure 4.

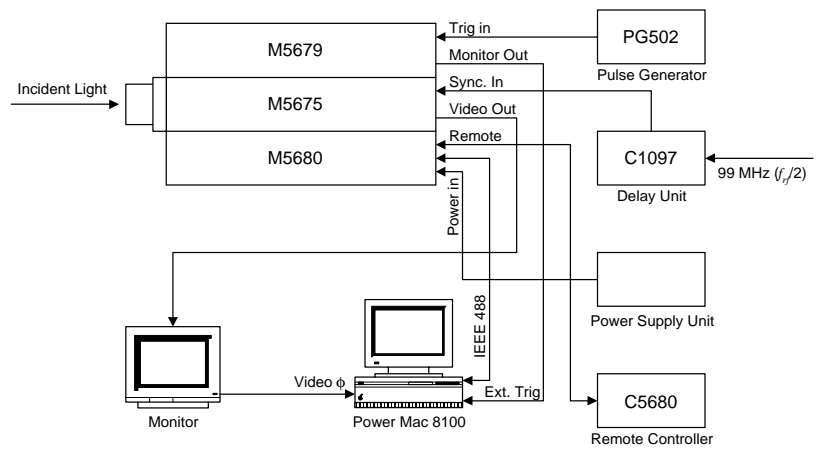

Figure 4: Configuration of the streak camera

The pulses of synchrotron radiation incident light being measured pass through the optics and enter the streak camera. The RF signal with the $99.76 \mathrm{MHz}$ frequency (half the RF frequency of the ring) goes into the M5675 as the vertical sweep timing signal after passing through the C1097. At the same time the pulse signal from the pulse generator PG502 acting as the horizontal sweep timing signal is sent to the M5679. The streak camera can be operated from the Power Macintosh 8100 computer with an image processing board, an GPIB board and the streak camera control software U5565. It can also be operated from the remote control panel. The video signal of the bunch image is monitored by a monitor and can be captured by the PC. 
The incident light pulses, only those bombard on the photocathode, are converted into electrons, and are effective for the streak camera measurement. The photocathode has an area of $150 \mu \mathrm{m}$ in height and $6 \mathrm{~mm}$ in width. The diaphragm before the camera lens can be chosen from several holes with the diameters of $10 \mu \mathrm{m}, 30 \mu \mathrm{m}$ and 100 $\mu \mathrm{m}$. Even if the diaphragm is opened completely, we must adjust the light being measured within the size of the photocathode. The light adjustment is one of the most important but difficult jobs for the bunch length measurement.

Figure 5 shows the bunch image of the BEPC storage ring we have obtained with only one electron bunch in the ring. The revolution period of the bunch in the storage ring is $801 \mathrm{~ns}$ and the horizontal sweep time of the streak camera is $5 \mathrm{~ms}$, so 6 consecutive turns of the same bunch can be seen on the screen at a time.

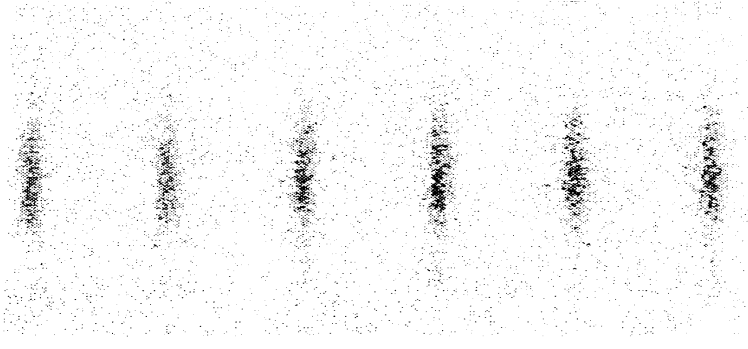

Figure 5: Beam image of 6 consecutive turns of the same bunch

The software U5565 gives the counts for each of 480 channels (or 480 time intervals) of the full scale. The rms bunch length $\sigma$ is obtained by fitting these data.

\section{POSITION SENSING PHOTO-DETECTOR}

As mentioned in the previous section, the beam position is measured with a single focusing lens system. After reflection from the three mirrors and passage through an $832 \mathrm{~mm}$ focusing lens, the image of the electron beam cross section is formed on a position sensing photodetector Model SC/10D of United Detector Technology (UDT). The SC/10D is the planer diffused construction PIN diode which provides the high performance and reliability with the low noise value for the $10 \times 10 \mathrm{~mm}^{2}$ active area.

For one of the two transverse planes, the two opposite output signals of the SC/10D are fed to a sum and difference amplifier Model 301B/AC of UDT. The sum and difference signals of the $301 \mathrm{~B} / \mathrm{AC}$ are digitized by a PCbased ADC card which fits into one ISA/EISA expansion slot in the backplane of an industry-standard compatible PC. The ADC has a 12-bit resolution and 25- $\mu$ s AD conversion time. The range of the sum signal is around 1-10 volts when the beam intensity is $10-100 \mathrm{~mA}$ in the case of the dedicated synchrotron radiation operating mode with the beam energy of $2.2 \mathrm{GeV}$. The difference signal is divided by the sum signal in the computer to obtain the normalized position signal of the light beam.
The normalized position signal versus the image position is calibrated by moving the SC/10D with a micrometer. As shown in Figure 6, the sensitivity of the light beam position is $0.467 \mathrm{~mm}$ in the central $50 \%$ active area of the SC/10D. Considering the magnification coefficient 0.139 of the optics, the sensitivity of the electron beam position is $3.36 \mathrm{~mm}$.

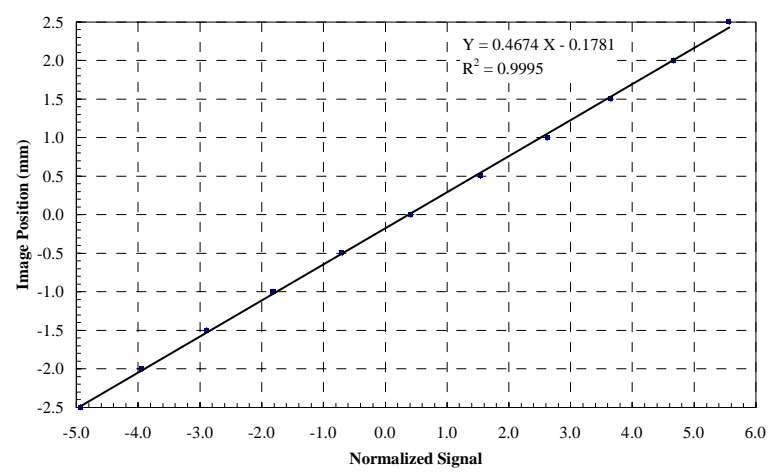

Figure 6: The sensitivity of the light beam position

Figure 7 shows a long term electron beam position drift of 60 hours. The data are stored in the BEPC archive database with the sampling time interval of 60 seconds.

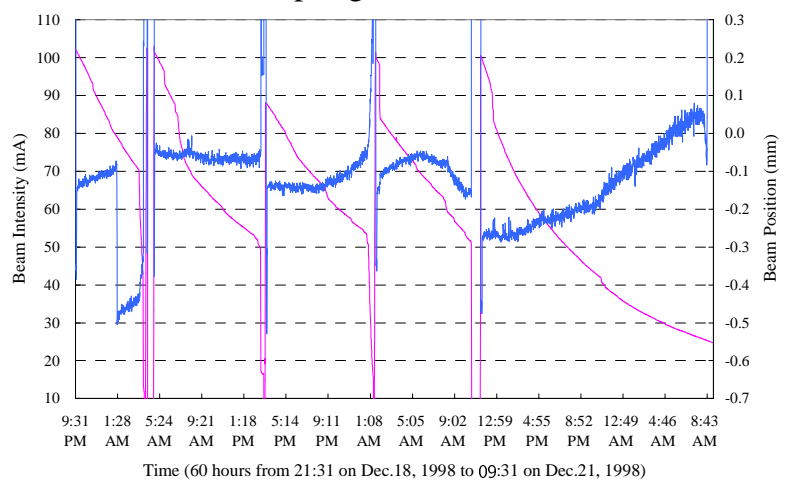

Figure 7: The beam position and beam intensity vs. time

\section{SUMMARY}

The synchrotron radiation monitor plays an important role in the BEPC beam diagnostic instrumentation system. Two new optical tables have been put into use and almost all measurement systems have been modified during the period from year 1996 to 1998. These modifications are very useful for overall machine operation and machine studies. The efforts are still carrying on to make measurements more accurate and reliable.

\section{ACKNOWLEDGMENTS}

The authors would like to thank all colleagues, especially Dr. Q.Zhang, who made contributions to build and upgrade the BEPC synchrotron radiation monitoring system.

\section{REFERENCES}

[1] D.Liu, L.Ma and K.Xue, "The Bunch Length Measurement of BEPC with a Streak Camera", Proceedings of ICEMI'97, Beijing, October 1997 